\title{
Interleukin-17 inhibits development of malignant pleural effusion via interleukin-9-dependent mechanism
}

\author{
Yong $\mathrm{Lu}^{1}$, Hua Lin ${ }^{2}$, Kan Zhai ${ }^{1}$, Xiaojuan Wang ${ }^{1}$, Qiong Zhou ${ }^{2 *} \&$ Huanzhong Shi ${ }^{1 * *}$ \\ ${ }^{1}$ Department of Respiratory and Critical Care Medicine, Beijing Chaoyang Hospital, Capital Medical University, Beijing 100020, China; \\ ${ }^{2}$ Department of Respiratory and Critical Care Medicine, Union Hospital, Tongji Medical College, Huazhong University of Science and Tech- \\ nology, Wuhan 430022, China
}

Received June 26, 2015; accepted July 5, 2016; published online August 12, 2016

\begin{abstract}
Th17 and Th9 cells have been demonstrated to possess immune regulatory functions in malignant pleural effusion (MPE). However, whether IL-17 can affect differentiation and function of Th9 cells in MPE remains unknown. The objective of the present study was to explore the impact of IL-17 on the in vivo differentiation of Th9 cells in relation to Th2 cells in a murine model of MPE, and to explore whether IL-17 inhibits MPE formation via IL-9-dependent mechanism. It was found that Th9 and Th2 cells were decreased in MPE from $I L-17^{-1-}$ mice as compared with wild type mice. IL-17 deficiency inhibited Th9 and Th2 cell differentiation via suppressing transcription factors IRF4 and GATA-3, respectively. IL-17 deficiency enhanced MPE formation by promoting angiogenesis and proliferation of pleural tumors, and thus accelerated the death of mice bearing MPE. The in vivo administration of anti-IL-9 neutralizing mAb accelerated the death of WT mice; whereas administration of exogenous IL-9 improved the survival of $I L-17^{-1-}$ mice. Our data provide the first definitive evidence that IL-17 promotes the differentiation of Th9 and Th2 cells in MPE. Our findings also demonstrate that IL-17 inhibits the formation of MPE and improves the survival of mice bearing MPE via an IL-9-dependent mechanism.
\end{abstract}

malignant pleural effusion, interleukin 17, Th9 cells

Citation: $\quad$ Lu, Y., Lin, H., Zhai, K., Wang, X., Zhou, Q., and Shi, H. (2016). Interleukin-17 inhibits development of malignant pleural effusion via interleukin-9-dependent mechanism.. Sci China Life Sci 59, 1297-1304. doi: 10.1007/s11427-016-0097-y

\section{INTRODUCTION}

IL-9, a member of the IL-2 cytokine family, is produced by naïve $\mathrm{CD} 4^{+} \mathrm{T}$ lymphocytes in response to IL- 4 and TGF- $\beta$ (Dardalhon et al., 2008; Veldhoen et al., 2008). IL-9 has been suggested to possess a functional role in some malignant lymphomas, such as Hodgkin's disease and large-cell anaplastic lymphoma (Fischer et al., 2003; Merz et al., 1991). It has been demonstrated that IL-9 plays a role during tumor genesis because dysregulated IL-9 response results in autonomous cell growth and malignant transformation of lymphoid cells associated with constitutive acti-

*Corresponding author (email: shihuanzhong@sina.com) **Corresponding author (emails: zhouqiongtj@126.com) vation of the Jak/STAT pathway (Knoops and Renauld, 2004).

Malignant pleural effusion (MPE) is a frequent and clinically significant systemic manifestation of various malignancies that adversely affects patient survival and quality of life (Kastelik, 2013; Roberts et al., 2010). It has been well documented that $\mathrm{CD}^{+} \mathrm{T}$ cell dominance occurs in MPE. Naïve $\mathrm{CD}^{+} \mathrm{T}$ cells become activated and differentiate into effector $\mathrm{T}$ helper (Th) cell subgroups, depending on the antigens and cytokine microenvironment they encounter. Our previous studies have demonstrated that several subgroups of $\mathrm{CD}^{+} \mathrm{T}$ cells, such as regulatory $\mathrm{T}$ cells (Tregs) (Chen et al., 2005), IL-17-producing $\mathrm{CD} 4^{+} \mathrm{T}$ cells (Th17 cells) (Ye et al., 2010), IL-22-producing $\mathrm{CD}^{+} \mathrm{T}$ cells (Th22 cells) 
(Ye et al., 2012a), and IL-9-producing CD4 ${ }^{+} \mathrm{T}$ cells (Th9 cells) (Ye et al., 2012b), play important immune regulatory roles in the pathogenesis of human MPE.

The relationship between Th9 and Th17 cells is of particular interest, and their immune regulation is often linked to proinflammatory and malignant processes. IL-9 can be produced by Th17 cells, which secrete mainly IL-17A and IL-17F (Beriou et al., 2010). On the other hand, IL-9 is able to induce Th17 cells to differentiate (Elyaman et al., 2009), and acts as a mediator of Th17 cell-associated inflammation and autoimmune process (Nowak et al., 2009; Singh et al., 2013). Our previous studies revealed that both Th17 and Th9 cells are increased in MPE which is promoted by local proinflammatory cytokines, and these Th cells exert an important immune regulation on lung cancer cells in tumor environment (Ye et al., 2010, 2012b). More recently, we have demonstrated that IL-17 deficiency promotes angiogenesis and proliferation activity of pleural tumors as well as pleural vascular permeability, and thus promotes MPE formation in the animal models of MPE using $I L-17^{-1-}$ mice (Lin et al., 2014). In the present study, we were prompted to explore the impact of IL-17 on the differentiation of Th9 cells in relation to Th2 cells in a murine model of MPE, and to explore whether IL-17 inhibits MPE formation via IL-9-dependent mechanism.

\section{RESULTS}

\section{IL-17 inhibited MPE formation}

In the previous study (Lin et al., 2014), we have demonstrated that more pleural tumor foci and bloody MPE are found in $I L-17^{-1-}$ mice as compared with WT mice 14 days after intrapleural injection of LLC cells. Here once again we confirmed that radiotracer uptake was significantly increased on FDG-PET scanning in $I L-17^{-1-}$ mice (Figure 1A). Furthermore, we found in the current study for the first time that the maximum standardized uptake value $\left(\mathrm{SUV}_{\max }\right)$ in WT mice was much lower than that in WT mice injected intraperitoneally with anti-IL-17 neutralizing $\mathrm{mAb}$ $(P=0.046)$; and that $\mathrm{SUV}_{\max }$ in $I L-17^{-1-}$ mice injected intraperitoneally with rmIL-17 was much lower than that in $I L-17^{-1-}$ mice $(P<0.001)$ (Figure $1 \mathrm{~A}$ and $\left.\mathrm{B}\right)$. These data indicate that both endogenous and exogenous IL-17 are capable of inhibiting the development of MPE.

As reported in our previous paper (Lin et al., 2014), expressions of both CD34 and $\mathrm{Ki}-67$ in pleural tumors are increased in $I L-17^{-1-}$ mice. We extended these work and further observed that intraperitoneal injection of anti-IL-17 $\mathrm{mAb}$ in WT mice significantly increased the numbers of both $\mathrm{CD} 4^{+}$(Figure $2 \mathrm{~A}$ ) and $\mathrm{Ki}-67^{+}$cells $(P<0.01)$ (Figure $2 \mathrm{~B})$; while intraperitoneal injection of rmIL-17 in $\mathrm{IL}-17^{-1-}$ mice significantly decreased the numbers of both $\mathrm{CD} 34^{+}$ and $\mathrm{Ki}-67^{+}$cells $(P<0.01)$.
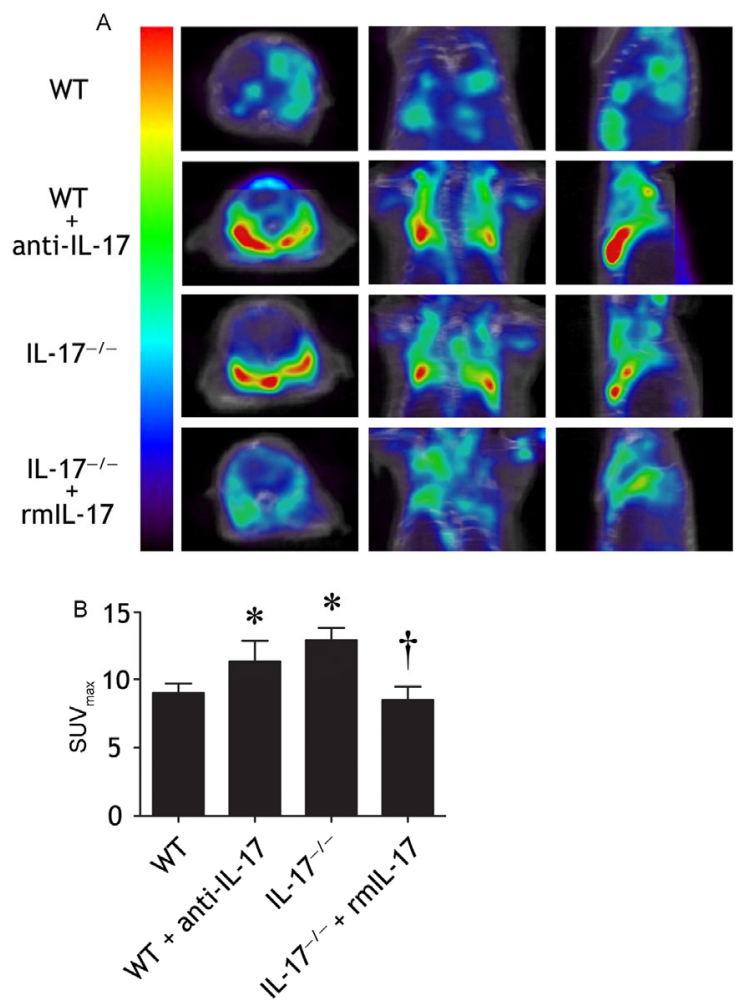

Figure 1 Effect of IL-17 on development of malignant pleural effusions (MPE). A, Fourteen days after intrapleural injection of Lewis lung cancer cells, positron emission tomography and computed tomography imaging of developing pleural tumors and MPE in wild type (WT) mice (top panels), WT mice receiving intraperitoneal injection of anti-IL-17A mAb (WT+anti-IL-17, second panels), $I L-17^{-1-}$ mice (third panels), and $I L-17^{-1-}$ mice receiving intraperitoneal injection of recombinant mouse IL-17A (IL-17 $17^{-1-}+$ rmIL-17, bottom panels). B, Comparison of maximum standardized uptake value $\left(\mathrm{SUV}_{\max }\right)$ on positron emission tomography were calculated among the above four groups (each $n=10$ ). Data are presented as means \pm SE. *,$P<0.05$ compared with WT group; $\dagger, P<0.001$ compared with $I L-17^{-1-}$ group, the comparisons were determined by one-way ANOVA.
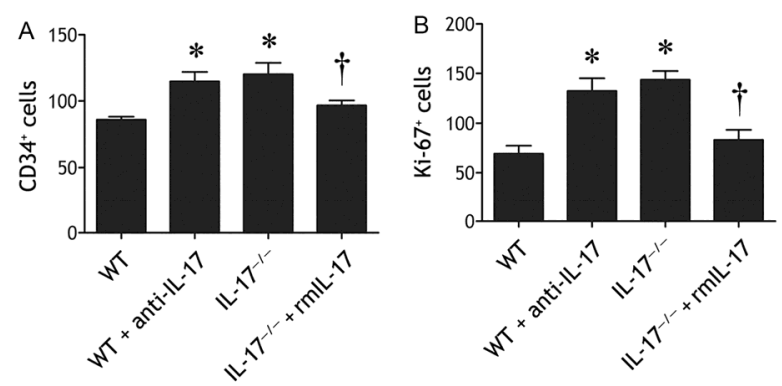

Figure 2 Effect of IL-17 on expression of CD34 and Ki-67 in pleural malignant tumor tissues. Tumor tissues were harvested from wild type (WT), WT mice receiving intraperitoneal injection of anti-IL-17 $\mathrm{mAb}$ (WT+anti-IL-17), $I L-17^{-1-}$ mice, and $I L-17^{-1-}$ mice receiving intraperitoneal injection of recombinant mouse IL-17A $\left(I L-17^{-1-}+\right.$ rmIL-17), the paraffin sections were stained with Abs specific to CD34 and Ki-67, 4',6-diamidino-2-phenylindole mounting medium was used for cell nuclei staining. Comparisons of $\mathrm{CD} 34^{+}$cells (A) and $\mathrm{Ki}-67^{+}$cells $(\mathrm{B})$ among the above four groups (each $n=5$ ) are shown. Data are presented as means \pm SE. *, $P<0.01$ compared with WT group; $\dagger, P<0.01$ compared with $I L-17^{-1-}$ group, the comparisons were determined by one-way ANOVA. 


\section{Th9 and Th2 cells were significantly increased in MPE}

We next investigated the distribution of Th9 cells in relation to Th2 cells in MPE using flow cytometry. In WT mice, a significant increase in Th9 cells (Figure 3A and B) and Th2 cells (Figures 3A and 2C) was observed in MPE, compared with those in the corresponding blood and spleen $(P<0.01)$. More importantly, we noted that the numbers of Th9 and Th2 cells were decreased in MPE from $I L-17^{-1-}$ mice as compared with those from WT mice $(P<0.001)$. Moreover, intraperitoneal injection of anti-IL-17 $\mathrm{mAb}$ decreased the numbers of MPE Th9 and Th2 cells in WT mice $(P<0.001)$. In addition intraperitoneal injection of rmIL-17 increased the numbers of MPE Th9 and Th2 cells in $I L-17^{-1-}$ mice $(P<0.001)$.

However, the numbers of Th9 and Th 2 cells in blood and spleen showed no significant differences in either $I L-17^{-1-}$ or WT mice $(P>0.05)$.

\section{Expressions of IL-9, IL-4, IRF4, and GATA-3 mRNA in MPE}

The mRNA expression of IL-9 (Figure 4A) and IL-4 (Figure 4B) was much lower in MPE from $I L-17^{-1-}$ mice than those from WT mice $(P<0.01)$. The intraperitoneal injection of anti-IL-17 mAb in WT mice significantly decreased
mRNA expression of IL-9 and IL-4, whereas the intraperitoneal injection of rmIL-17 in $I L-17^{-1-}$ mice significantly increased mRNA expression of IL-9 and IL-4 $(P<0.01)$. In parallel to the change in mRNA expression of IL-9 and IL-4, very similar change patterns in mRNA expression of IRF4 (Figure 4C) and GATA-3 (Figure 4D) were also observed $(P<0.01)$.

\section{IL-17 affects survival of mice bearing MPE via IL-9-dependent mechanism}

The median survival times of WT, WT+anti-IL-9, $I L-17^{-1-}$, and $I L-17^{-1-}+$ rmIL-9 mice bearing MPE were 26.0, 19.0, 20.0, and 26.0 days, respectively. The overall log rank test for survival among these four groups was significant $(P<0.001)$. Pair-wise log rank tests showed that the survival of $I L-17^{-1-}$ mice was much shorter than that of WT mice $(P<0.001)$. Intraperitoneal injection of anti-IL-9 mAb accelerated the death of WT mice $(P<0.001)$, whereas intraperitoneal injection of rmIL-9 improved the survival of $I L-17^{-1-}$ mice $(P<0.001)$ (Figure 5). The survival of the two control groups (isotype mAb control for WT+anti-IL-9 group and PBS containing $0.1 \%$ albumin control for $I L-17^{-1-}+$ rmIL-9 group) was similar to that of WT mice (data not shown).
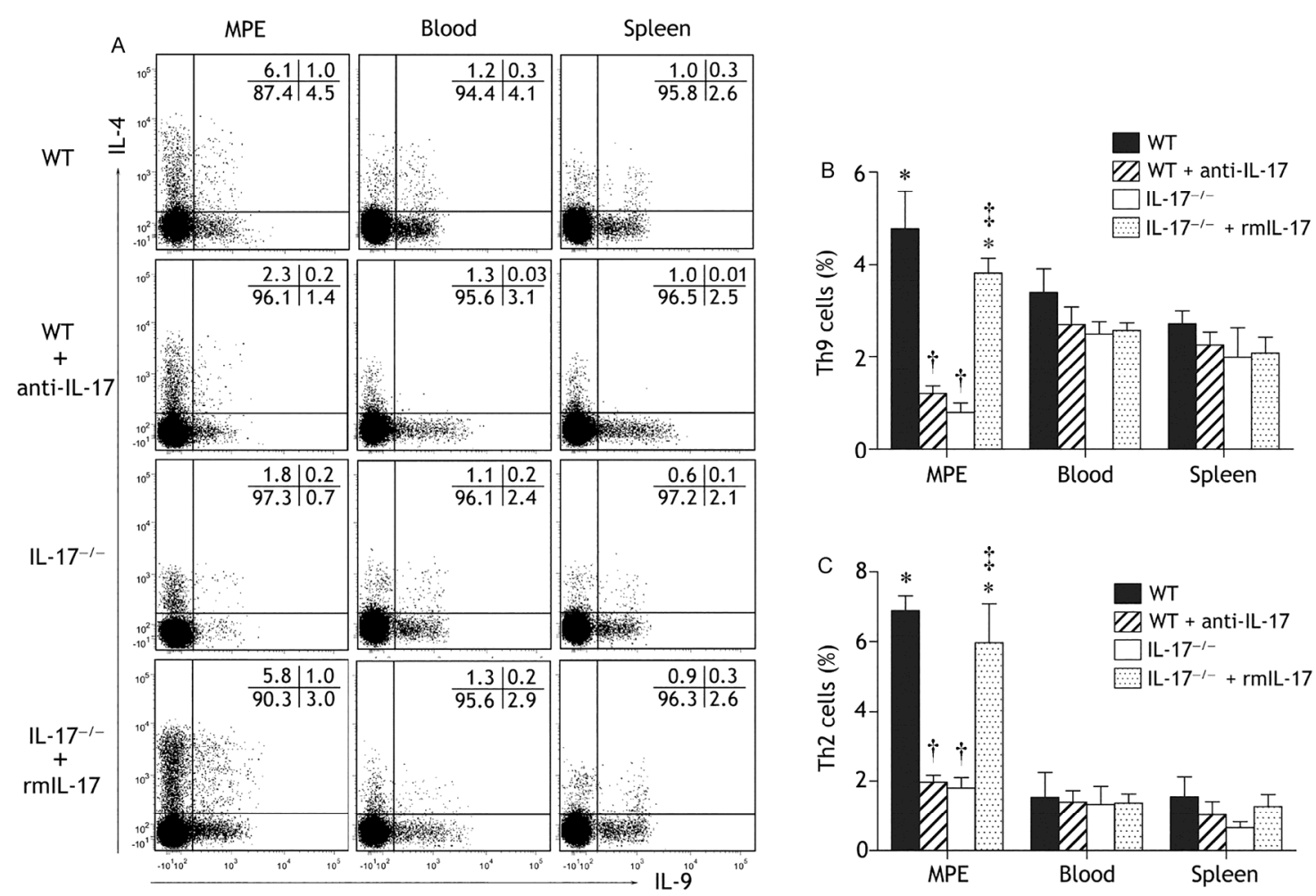

Figure 3 Effect of IL-17 on distributions of Th9 and Th2 cells in malignant pleural effusions (MPE), blood and spleen. A, The representative flow cytometric dot plots of Th9 and Th2 cells in wild type (WT) mice (top panels), WT mice receiving intraperitoneal injection of anti-IL-17 mAb (WT+anti-IL-17, second panels), $I L-17^{-1}$ mice (third panels), and $I L-17^{-1-}$ mice receiving intraperitoneal injection of recombinant mouse IL-17 (IL-1 $17^{-1}+$ rmIL-17, bottom panels). Comparisons of percentages of Th9 (B) and Th2 cells (C) in MPE, blood and spleen from the above four groups (each $n=10$ ). Data are presented as means \pm SE. *, $P<0.01$ compared with blood and spleen, the comparisons were determined by two-way ANOVA. $\dagger, P<0.001$ compared WT mice; $\ddagger, P<0.001$ compared with $I L-17^{-1-}$ mice, the comparisons were determined by one-way ANOVA. 

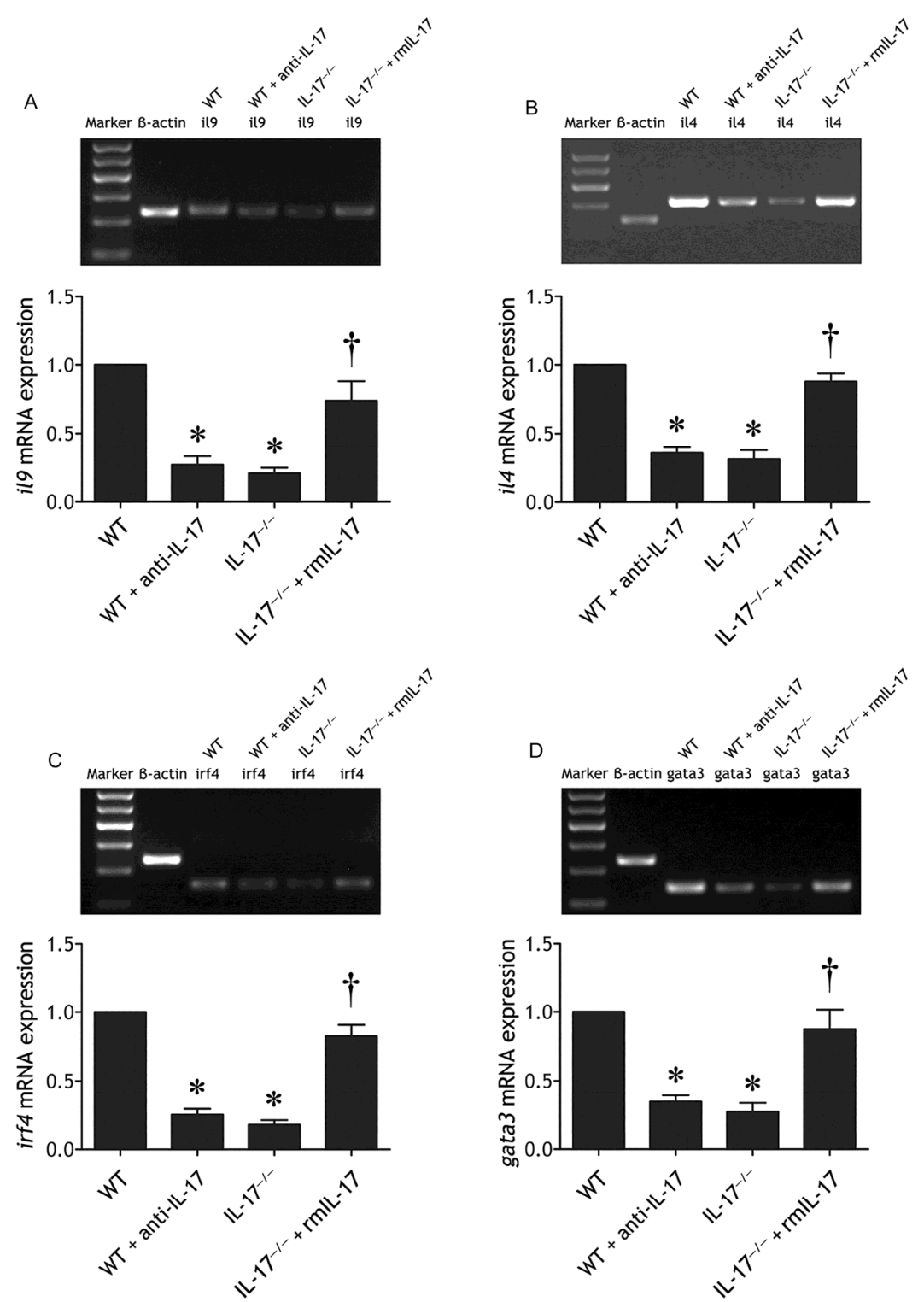

Figure 4 mRNA expression of IL-9 and IL-4 and their corresponding transcription factors in malignant pleural effusions (MPE). The mononuclear cells were isolated from MPE of wild type (WT) mice (top panels), WT mice receiving intraperitoneal injection of anti-IL-17 mAb (WT+anti-IL-17, second panels), $I L-17^{-1-}$ mice (third panels), and $I L-17^{-1-}$ mice receiving intraperitoneal injection of recombinant mouse IL-17 (IL-1 $17^{-1-}+$ rmIL-17, bottom panels). RNA was isolated, and qPCR was performed for il9 mRNA (A), il4 mRNA (B), irf4 mRNA (C), and gata3 mRNA (D). Electrophoresis' photographs are representatives of four independent experiments. Data are presented as mean $\pm \mathrm{SE}$ of four experiments. *, $P<0.01$ compared with WT mice; $\dagger, P<0.01$ compared with $I L-17^{-1-}$ mice, the comparisons were determined by one-way ANOVA.

\section{DISCUSSION}

In the presence of TGF- $\beta 1$, IL-9 differentiates naïve $\mathrm{CD} 4^{+}$ T cells into Th17 cells; IL-9 also enhances the suppressive functions of Tregs in vitro. The mechanism by which IL-9 affects Th17 cells and Tregs is through activation of STAT3 and STAT5 signaling (Elyaman et al., 2009). Singh and colleagues have shown that IL-9 has a role in the development of psoriatic lesions through Th17 cell-associated inflammation and angiogenesis (Singh et al., 2013). Zhou and colleagues have reported hat IL-9 induces chemokine CCL-20 production by astrocytes to enhance the infiltration of Th17 cells into the central nervous system (Zhou et al., 2011). To the best of our knowledge, the current study was the first one to explore the impact of IL-17 on the differentiation and immune regulation of Th9 cells.

The contribution of IL-17 or Th17 cells to tumor immunity has been well documented (Ye et al., 2013; Zou and Restifo, 2010). Our previous studies showed that Th17 cell numbers in MPE are significantly increased, and that overrepresentation of Th17 cells in MPE may be due to Th17 cell differentiation and expansion stimulated by local proinflammatory cytokines, and to recruitment of Th17 cells from blood induced by pleural chemokines CCL20 and 


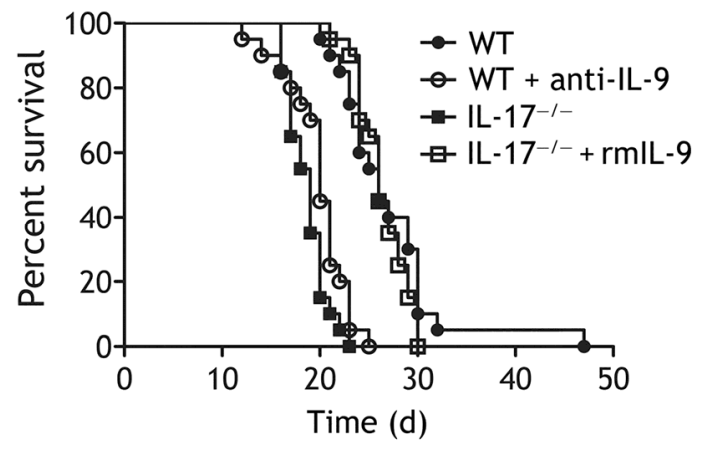

Figure 5 IL-17 improved survival of mice bearing malignant pleural effusion by affecting Th9 response. Mice ( 15 mice per group) were administered intrapleural injection of Lewis lung cancer cells. Life span analysis showed that $I L-17^{-1-}$ group had a significant shorter survival than wild type (WT) group; intraperitoneal injection of WT mice with anti-IL-9 mAb (WT+anti-IL-9) had a significant shorter survival than WT group; and intraperitoneal injection of recombinant mouse IL-9 (IL-17 $\left.{ }^{-1-}+\mathrm{IL}-9\right)$ had a significant improved survival compared with $I L-17^{-1-}$ mice. Overall survival was estimated by the Kaplan-Meier method and compared by the log-rank test.

CCL22 (Ye et al., 2010). On the other hand, Tregs inhibit the generation and differentiation of Th17 cells via a latency-associated peptide-dependent mechanism (Ye et al., 2011). More recently, we have demonstrated that IL-17 deficiency promotes angiogenesis and proliferation activity of pleural tumors as well as pleural vascular permeability, and thus promotes MPE formation (Lin et al., 2014). The finding that IL-17 deficiency accelerates the death of mice bearing MPE observed in the animal study (Lin et al., 2014) is consistent with the finding that the accumulation of Th17 cells in MPE predicts improved patient survival in the human study (Ye et al., 2010). Consistent with our previous findings (Lin et al., 2014), we confirmed that IL-17 deficiency promoted MPE formation by enhancing the angiogenesis and proliferation of pleural tumors, and that in vivo administration of anti-IL-17 mAb in WT mice was able to promote MPE formation by increasing tumor angiogenesis and proliferation, while in vivo administration of rmIL-17 in $I L-17^{-1-}$ mice was able to inhibit MPE formation by diminishing tumor angiogenesis and proliferation.

Previous studies have suggested that IL-9 plays an important role in NK/T-cell lymphoma possibly via an autocrine mechanism (Nagato et al., 2005). Purwar and colleagues (Purwar et al., 2012) have demonstrated that that mice deficient in Th17 cell development have substantial resistance to melanoma growth in a IL-9-dependent manner; and treatment with exogenous IL-9 inhibits tumor growth in vivo. $\mathrm{Lu}$ and colleagues have found that tumor-infiltrating Th9 cells induce lung tumor tissues, especially epithelial cells, to express CCL20, which recruits inflammatory cells, especially dendritic cells, into tumor sites, and subsequently promotes a strong activation response of $\mathrm{CD}^{+}$tumor-infiltrating lymphocytes (Lu et al., 2012). These data suggest a distinct role for tumor-specific Th9 cells in pro- voking $\mathrm{CD}^{+} \mathrm{T}$ cell-mediated anti-tumor immunity. On the other hand, intratumor Th2 cells have been shown to correlate with cancer-associated fibroblast thymic stromal lymphopoietin production and to reduce survival of cancer patients (De Monte et al., 2011). The relationship between Th9 cells and Th2 cells is of particular interest, and their immune regulation is often linked to proinflammatory processes and tumor immunity. We therefore explored the distribution of Th9 cells in relation to Th2 cells in MPE and found that the numbers of both Th9 and Th2 cells in MPE were significantly higher than those in blood and spleen.Nevertheless the numbers of Th9 and Th2 cells were significantly lower in MPE, but not in either spleen or blood, from $I L-17^{-1-}$ mice than those from WT mice, indicating that the differentiation of Th9 and Th2 cells in MPE can be promoted by IL-17 in vivo.

Since IRF4 is a transcription factor that is essential for the developmental program of Th9 cells (Staudt et al., 2010; Tamiya et al., 2013), and the differentiation of Th2 cell lineage requires the upregulation of GATA-3 (Zheng and Flavell, 1997; Zhou and Ouyang, 2003), we thus examined mRNA expression of IL-9 and IL-4 simultaneously with mRNA expression of IRF4 and GATA-3, and found that there was much lower mRNA expression of IL-9 and IRF4 in MPE from $I L-17^{-1-}$ mice than that from WT mice. Similarly, mRNA expression of IL-4 and GATA-3 was also lower in MPE from $I L-17^{-1-}$ mice than that from WT mice. Collectively, our data suggested that IL-17 enhances differentiation Th9 and Th2 cells by up-regulating expression of IRF4 and GATA-3, respectively.

Our previous data showed that IL-9 significantly promotes the proliferation response and migratory activity of lung cancer cells by activating STAT3 signaling, and that IL-9 strongly promotes intercellular adhesion of lung cancer cells to pleural mesothelial cells by upregulating the expression of cellular adhesion molecules ICAM-1, LFA-1 and VCAM-1 on both cancer cells and pleural mesothelial cells (Ye et al., 2012b). All these functional aspects of IL-9 provide the explanation for our observation that the accumulation of Th9 cells in MPE predicts reduced patient survival, with increased risk for death in MPE patients (Ye et al., $2012 b$ ). In the present study, we noted a significant correlation between IL-17 deficiency and mouse survival. The absence of IL-17 was a significant predictor for short survival of MPE mice, intraperitoneal injection of anti-IL-9 neutralizing $\mathrm{mAb}$ accelerated the death of WT mice bearing MPE, and intraperitoneal injection of rmIL-9 improved the survival of $I L-17^{-1-}$ mice bearing MPE. Our data indicate that IL-17 reduces the death of mice bearing MPE via IL-9-dependent mechanism.

In conclusion, we have demonstrated that both $\mathrm{Th} 9$ and Th2 cells were decreased in MPE from $I L-17^{-1-}$ mice as compared with WT mice. IL-17 inhibits MPE formation by attenuating angiogenesis and proliferation of pleural tumor, 
and thus reduces the death of mice bearing MPE via IL-9-dependent mechanism. Thus, therapeutic intervention aiming at interfering Th17 or/and Th9 cells may be beneficial for improving prognosis in patients with MPE.

\section{MATERIALS AND METHODS}

\section{Animals}

Wild type (WT) C57BL/6 mice were purchased from the Animal Center of Wuhan University (Wuhan, China). IL-17 $a^{-1-}$ mice in a C57BL/6 background were generated as previously described (Nakae et al., 2002) and were provided by Dr. Iwakura (University of Tokyo, Japan). Mice used for experiments were sex-, weight (20-25 g)-, and age (8-12 wk)-matched. The mice were maintained on a chow diet in a 12-hour light/12-hour dark environment at $25^{\circ} \mathrm{C}$ in Animal Care Facility of Beijing Chaoyang Hospital or in Tongji Medical College Animal Care Facility, according to institutional guidelines. All animal studies were approved by Institutional Animal Care and Utilization Committees of Beijing Chaoyang Hospital, Capital Medical University and Tongji Medical College, Huazhong University of Science and Technology.

\section{MPE Model}

A mouse model of MPE by intrapleural injecting Lewis lung cancer (LLC) cells was made as described previously (Lin et al., 2014). Briefly, mice were anesthetized by isoflurane inhalation, the skin overlying the anterior and lateral chest wall was shaved and disinfected, and a 5-mm-long trans-verse skin incision was made on the right anterolateral thoracic area at the xiphoid level. Fascia and muscle were retracted, and $1.5 \times 10^{5}$ LLC cells (purchased from the American Type Culture Collection (Manassas, USA) suspended in $50 \mu \mathrm{L}$ phosphate buffered saline (PBS) were injected into pleural cavity through an intercostal space under direct observation. The skin incision was closed using continuous 5-0 Ethilon monofilament suture, and the animals were monitored until complete recovery.

\section{Sample collection and processing}

Fourteen days after pleural injection of LLC cells, mice were euthanized by $\mathrm{CO}_{2}$ asphyxiation, and blood was drawn from the retro-orbital veins. Thereafter, the abdominal wall was opened, and the viscera were retracted to visualize the diaphragm. Pleural fluid was gently aspirated using a 3-mL syringe, and its volume was measured with a $1,000-\mu \mathrm{L}$ pipette. Smear slides were made from samples for WrightGiemsa staining and were determined for leukocyte differential counts under light microscope.

MPE and blood samples from each mouse were collected in heparin-treated tubes, and were immersed in ice immediately and were then centrifuged at $300 \times g$ for $10 \mathrm{~min}$. In order to obtain spleen cell suspension, spleens were removed and cut into small pieces, and then minced in PBS and filtered. The cell pellets of MPE, blood, and spleen were resuspended in PBS, and mononuclear cells were isolated by Ficoll-Hypaque gradient centrifugation (Pharmacia, Sweden) to determine $\mathrm{T}$ cell subsets within $1 \mathrm{~h}$.

\section{In vivo treatment of mice}

At days 1, 3, 6, 9, and 12 after intrapleural instillation of LLC cells, WT mice $(n=10)$ received intraperitoneal injection of $100 \mu \mathrm{g}$ anti-IL-17A neutralizing mAb (clone eBioMM17F3, eBioscience, $\operatorname{lgG} 1$, USA) or $100 \mu \mathrm{g}$ mouse IgG1 isotype control mAb (eBioscience); while $I L-17^{-1-}$ mice were injected intraperitoneally at a dose of $1 \mu \mathrm{g}$ recombinant mouse (rm) IL-17A diluted in PBS containing $0.1 \%$ albumin (R\&D System, USA) or PBS containing $0.1 \%$ albumin in a total volume of $100 \mu \mathrm{L}$. The administration regimens of anti-IL-17 mAb and rmIL-17 were based upon our preliminary experiments.

\section{Positron emission tomography (PET) and computed tomography (CT) scanning}

All PET images were acquired using Trans-PET ${ }^{\circledR}$ BioCaliburn $^{\text {TM }}$ LH (Raycan Technology Co., Ltd, Suzhou) consisting of 12 basic detector modules. Fifteen-min PET scans were begun between 60 and 70 min after retro-orbital injection of 200-300 $\mu \mathrm{Ci}$ of 18-fluoro-6-deoxy-glucose. In consideration of the PET system's spatial resolution, reconstructed PET images had pixel dimensions of $(0.5 \mathrm{~mm})^{2}$ and $0.5 \mathrm{~mm}$ slice thickness. Furthermore, due to the OSEM reconstruction method using point spread function system response matrix, the size of the reconstructed $3 \mathrm{D}$ image was set to be $280 \times 280 \times 104(X \times Y \times Z)$ that must be smaller than or equal to the size of point spread function system response matrix. Also, CT images were acquired on a small animal CT scanner provided by the Trans-PET system with characteristics of an axial field of view of $5.0 \mathrm{~cm}$, a radial field of view of $7.0 \mathrm{~cm}$ and a spatial resolution of $0.5 \mathrm{~mm}$. The PET and CT images were acquired with the mouse under anesthesia in a common animal holder. The co-registration of the images was implemented using the AMIDE software package (The Free Software Foundation Inc., USA).

\section{Immunofluorescence staining}

Pleural tumor tissues were fixed in $4 \%$ paraformaldehyde, embedded with paraffin. The paraffin-embedded tissues were cut into 5- $\mu \mathrm{m}$ thick sections that were mounted on glass slides. The primary Abs were rat anti-mouse CD34 $\mathrm{mAb}$ (both were purchased from Abcam, UK) and rabbit $\mathrm{mAb}$ targeted against mouse $\mathrm{Ki}-67$, appropriate species-matched Abs were used as isotype controls. As secondary Abs, dylight 488-conjugated affinity-purified goat anti-rat IgG (EarthOx, USA) was used for labeling the rat anti-mouse $\mathrm{CD} 34 \mathrm{mAb}$, and cy3-conjugated affinity-purified goat anti-rabbit IgG (Proteintech Group Inc, USA) was used for labeling the rabbit anti-mouse Ki-67 Ab. The slides were processed and then incubated with $10 \%$ 
goat serum in PBS at room temperature for $30 \mathrm{~min}$, and then incubated at $4^{\circ} \mathrm{C}$ overnight with $1 / 500$ concentrations of primary Abs. After washing, slides were incubated with secondary Abs for $120 \mathrm{~min}$ at room temperature in the dark. 4',6-diamidino-2-phenylindole mounting medium (Vector Laboratories, USA) was used for cell nuclei staining. Finally, slides were examined under laser scanning confocal microscope (Olympus FV500; Olympus, Japan).

\section{Flow cytometry}

For intracellular detection of cytokines, cells were stimulated for $4 \mathrm{~h}$ at $37^{\circ} \mathrm{C}$ with $50 \mathrm{ng} \mathrm{mL} \mathrm{m}^{-1} \mathrm{PMA}$ (Sigma-Aldrich, USA) and $1 \mu \mathrm{g} \mathrm{mL}^{-1}$ ionomycin (Sigma-Aldrich) in the presence of Brefeldin A $\left(10 \mu \mathrm{g} \mathrm{mL}^{-1}\right.$, Enzo Life Science, USA). The Abs including anti-CD3, -CD8, -IL-4, -GATA-3, and -interferon-regulatory factor 4 (IRF4) mAbs were purchased from BDPharmingen (San Diego, USA) or eBioscience (San Diego). Cells were surface-stained with anti-CD3 and -CD8 $\mathrm{mAbs}$ in PBS+2\% heat-inactivated fetal bovine serum (FBS, Gibco, USA) for $20 \mathrm{~min}$ at $4{ }^{\circ} \mathrm{C}$. Cells were resuspended in a fixation/permeabilization solution (Cytofix/Cytoperm; BD Pharmingen, USA) and incubated with anti-IL-4, -IL-9, -GATA-3 or -IRF4 mAbs for $30 \mathrm{~min}$ at $4^{\circ} \mathrm{C}$. Cells were then washed with permeabilization buffer and resuspended in PBS+2\% FBS for flow cytometric analysis (FACSCanto II; BD Bioscience, USA). Flow cytometry was performed on a FACS Canto II (BD Biosciences) and analyzed using BD FCSDiva Software and FCS Express 4 software (De Novo Software, USA).

\section{Quantitative real time PCR}

Total RNA was isolated from cell pellets with Trizol reagent (Invitrogen, USA). cDNAs were synthesized with oligo (dT) primers using the First Strand cDNA Synthesis Kit ReverTra Ace- $\alpha$-(TOYOBO, Japan). cDNAs were used as templates in real time PCR with the SYBR Green I Real time PCR Master Mix (TOYOBO). DNA was amplified under the following typical cycling conditions: denaturation at $95^{\circ} \mathrm{C}$ for $15 \mathrm{~s}$, annealing at $58^{\circ} \mathrm{C}$ for $20 \mathrm{~s}$, extension at $72^{\circ} \mathrm{C}$ for $20 \mathrm{~s}$. The samples were amplified for 40 cycles. The following primers were used: for $i l 4$, forward primer: 5'-TGTCATCCTGCTCTTCTTTCTC-3', reverse primer: 5'-CACTCTCTGTGGTGTTCTTCGT-3'; for $i l 9$, forward primer: 5'-GGTGACATACATCCTTGCCTCT-3', reverse primer: 5'-CCCTCCCTGTAGCACGGT-3'; for gata3, forward primer: 5'-TTACCACCTATCCGCCCTA-3' reverse primer: 5'-ACACTCCCTGCCTTCTGTG-3'; for irf4, forward primer: 5'-CAATGTCCTGTGACGTTTGG-3', reverse primer: 5'-GGCTTCAGCAGACCTTATGC-3'; for $\beta$-actin, forward primer: 5'-CTGAGAGGGAAATCGTGCGT-3', reverse primer: 5'-CCACAGGATTCCATACCCAAGA-3'. $\beta$-actin was used as an internal control, and levels of each gene were normalized to $\beta$-actin expression using the $\Delta \Delta \mathrm{Ct}$-method, with WT mouse experimental value set to 1 . PCR products were also analyzed in $2 \%$ agarose gel electrophoresis.

\section{Survival advantage}

The observation of survival advantage included 3 groups of WT and 3 groups of $I L-17^{-1-}$ mice (each $n=15$ ). At days 1 , $3,6,9$, and 12 after intrapleural instillation of LLC cells, 2 groups of WT mice received intraperitoneal injection of 100 $\mu \mathrm{g}$ anti-IL-9 neutralizing mAb (clone D9302C12, eBioscience) (WT+anti-IL-9) or $100 \mu \mathrm{g}$ mouse isotype control $\mathrm{mAb}$ (eBioscience), respectively; and 2 groups of $I L-17^{-1}$ mice received intraperitoneal injection of $1 \mu \mathrm{g}$ rmIL-9 (R\&DSystem, USA) diluted in PBS containing $0.1 \%$ albumin $\left(I L-17^{\prime-}+\right.$ rmIL-9) or PBS containing $0.1 \%$ albumin in a total volume of $200 \mu \mathrm{L}$, respectively. One group of WT and 1 group of $I L-17^{-1-}$ mice receiving no intraperitoneal injections served as controls. The mice without heartbeat and breathing were determined to be dead. The doses of anti-IL-9 mAb and rmIL-9 were based upon our preliminary experiments.

\section{Statistics}

Data were normally distributed, and all values are expressed as mean \pm SE. Differences between two or multiple experimental groups were compared using Student's $t$ test, one-way analysis of variance (ANOVA), or two-way ANOVA, as appropriate. Survival was estimated by the Kaplan-Meier method and compared by the log-rank test. Analysis was completed with SPSS version 16.0 Statistical Software (USA), and $P<0.05$ was considered to indicate statistical significance.

Compliance and ethics The author(s) declare that they have no conflict of interest.

Acknowledgements This work was supported by the National Natural Science Foundation of China (91442109, 31470883, 81270149, 81272591), and the National Basic Research Program of China (2012CB518706).

Adamson, A.S., Collins, K., Laurence, A., and O'Shea, J.J. (2009). The Current STATus of lymphocyte signaling: new roles for old players. Curr Opin Immunol 21, 161-166.

Beriou, G., Bradshaw, E.M., Lozano, E., Costantino, C.M., Hastings, W.D., Orban, T., Elyaman, W., Khoury, S.J., Kuchroo, V.K., Baecher-Allan, C., and Hafler, D.A. (2010). TGF-beta induces IL-9 production from human Th17 cells. J Immunol 185, 46-54.

Chen, Y.Q., Shi, H.Z., Qin, X.J., Mo, W.N., Liang, X.D., Huang, Z.X., Yang, H.B., and $\mathrm{Wu}, \mathrm{C}$. (2005). $\mathrm{CD}^{+} \mathrm{CD} 25^{+}$regulatory T lymphocytes in malignant pleural effusion. Am J Respir Crit Care Med 172, 1434-1439.

Dardalhon, V., Awasthi, A., Kwon, H., Galileos, G., Gao, W., Sobel, R.A., Mitsdoerffer, M., Strom, T.B., Elyaman, W., Ho, I.C., Khoury, S., Oukka, M., and Kuchroo, V.K. (2008). IL-4 inhibits TGF-beta-induced Foxp $3^{+} \mathrm{T}$ cells and, together with TGF-beta, generates IL- $9^{+}$IL- $10^{+}$ Foxp3 ${ }^{-}$effector T cells. Nat Immunol 9, 1347-1355.

De Monte, L., Reni, M., Tassi, E., Clavenna, D., Papa, I., Recalde, H., Braga, M., Di Carlo, V., Doglioni, C., and Protti, M.P. (2011). 
Intratumor $\mathrm{T}$ helper type 2 cell infiltrate correlates with cancer-associated fibroblast thymic stromal lymphopoietin production and reduced survival in pancreatic cancer. J Exp Med 208, 469-478.

Elyaman, W., Bradshaw, E.M., Uyttenhove, C., Dardalhon, V., Awasthi, A., Imitola, J., Bettelli, E., Oukka, M., van Snick, J., Renauld, J.C. (2009). IL-9 induces differentiation of TH17 cells and enhances function of Foxp $3^{+}$natural regulatory T cells. Proc Natl Acad Sci USA $106,12885-12890$.

Fischer, M., Bijman, M., Molin, D., Cormont, F., Uyttenhove, C., van Snick, J., Sundstrom, C., Enblad, G., and Nilsson, G. (2003). Increased serum levels of interleukin-9 correlate to negative prognostic factors in Hodgkin's lymphoma. Leukemia 17, 2513-2516.

Goswami, R., Jabeen, R., Yagi, R., Pham, D., Zhu, J., Goenka, S., and Kaplan, M.H. (2012). STAT6-dependent regulation of Th9 development. J Immunol 188, 968-975.

Kaplan, M.H., Schindler, U., Smiley, S.T., and Grusby, M.J. (1996). Stat6 is required for mediating responses to IL-4 and for development of Th2 cells. Immunity 4, 313-319.

Kastelik, J.A. (2013). Management of malignant pleural effusion. Lung 191, 165-175.

Knoops, L., and Renauld, J.C. (2004). IL-9 and its receptor: from signal transduction to tumorigenesis. Growth Factors 22, 207-215.

Lin, H., Tong, Z.H., Xu, Q.Q., Wu, X.Z., Wang, X.J., Jin, X.G., Ma, W.L., Cheng, X., Zhou, Q., and Shi, H.Z. (2014). Interplay of Th1 and Th17 cells in murine models of malignant pleural effusion. Am J Respir Crit Care Med 189, 697-706.

Lu, Y., Hong, S., Li, H., Park, J., Hong, B., Wang, L., Zheng, Y., Liu, Z., Xu, J., He, J., Yang, J., Qian, J., Yi, Q. (2012). Th9 cells promote antitumor immune responses in vivo. J Clin Invest 122, 4160-4171.

Maier, E., Duschl, A., and Horejs-Hoeck, J. (2012). STAT6-dependent and -independent mechanisms in Th2 polarization. Eur J Immunol 42, 2827-2833.

Merz, H., Houssiau, F.A., Orscheschek, K., Renauld, J.C., Fliedner, A., Herin, M., Noel, H., Kadin, M., Mueller-Hermelink, H.K., and Van Snick, J. (1991). Interleukin-9 expression in human malignant lymphomas: unique association with Hodgkin's disease and large cell anaplastic lymphoma. Blood 78, 1311-1317.

Nagato, T., Kobayashi, H., Kishibe, K., Takahara, M., Ogino, T., Ishii, H., Oikawa, K., Aoki, N., Sato, K., Kimura, S., Shimizu, N., Tateno, M., Harabuchi, Y. (2005). Expression of interleukin-9 in nasal natural killer/T-cell lymphoma cell lines and patients. Clin Cancer Res 11, $8250-8257$.

Nakae, S., Komiyama, Y., Nambu, A., Sudo, K., Iwase, M., Homma, I., Sekikawa, K., Asano, M., and Iwakura, Y. (2002). Antigen-specific T cell sensitization is impaired in IL-17-deficient mice, causing suppression of allergic cellular and humoral responses. Immunity 17, 375-387.

Nowak, E.C., Weaver, C.T., Turner, H., Begum-Haque, S., Becher, B., Schreiner, B., Coyle, A.J., Kasper, L.H., and Noelle, R.J. (2009). IL-9 as a mediator of Th17-driven inflammatory disease. J Exp Med 206, $1653-1660$.

O'Shea, J.J., Lahesmaa, R., Vahedi, G., Laurence, A., and Kanno, Y. (2011). Genomic views of STAT function in $\mathrm{CD}^{+}{ }^{+} \mathrm{T}$ helper cell differentiation. Nat Rev Immunol 11, 239-250.
Purwar, R., Schlapbach, C., Xiao, S., Kang, H.S., Elyaman, W., Jiang, X., Jetten, A.M., Khoury, S.J., Fuhlbrigge, R.C., Kuchroo, V.K., Clark, R.A., and Kupper, T.S. (2012). Robust tumor immunity to melanoma mediated by interleukin-9-producing T cells. Nat Med 18, 1248-1253.

Roberts, M.E., Neville, E., Berrisford, R.G., Antunes, G., and Ali, N.J. (2010). Management of a malignant pleural effusion: British Thoracic Society Pleural Disease Guideline 2010. Thorax 65 Suppl 2, ii32-40.

Singh, T.P., Schon, M.P., Wallbrecht, K., Gruber-Wackernagel, A., Wang, X.J., and Wolf, P. (2013). Involvement of IL-9 in Th17-associated inflammation and angiogenesis of psoriasis. PLoS One 8, e51752.

Staudt, V., Bothur, E., Klein, M., Lingnau, K., Reuter, S., Grebe, N., Gerlitzki, B., Hoffmann, M., Ulges, A., Taube, C., Dehzad, N., Becker, M., Stassen, M., Steinborn, A., Lohoff, M., Schild, H., Schmitt, E., and Bopp, T. (2010). Interferon-regulatory factor 4 is essential for the developmental program of T helper 9 cells. Immunity 33, 192-202.

Tamiya, T., Ichiyama, K., Kotani, H., Fukaya, T., Sekiya, T., Shichita, T., Honma, K., Yui, K., Matsuyama, T., Nakao, T., Fukuyama, S., Inoue, H., Nomura, M., and Yoshimura, A. (2013). Smad2/3 and IRF4 play a cooperative role in IL-9-producing T cell induction. J Immunol 191, 2360-2371

Veldhoen, M., Uyttenhove, C., van Snick, J., Helmby, H., Westendorf, A., Buer, J., Martin, B., Wilhelm, C., and Stockinger, B. (2008). Transforming growth factor-beta "reprograms" the differentiation of T helper 2 cells and promotes an interleukin 9-producing subset. Nat Immunol 9, 1341-1346.

Ye, J., Livergood, R.S., and Peng, G. (2013). The role and regulation of human Th17 cells in tumor immunity. Am J Pathol 182, 10-20.

Ye, Z.J., Zhou, Q., Gu, Y.Y., Qin, S.M., Ma, W.L., Xin, J.B., Tao, X.N., and Shi, H.Z. (2010). Generation and differentiation of IL-17-producing $\mathrm{CD}^{+} \mathrm{T}$ cells in malignant pleural effusion. J Immunol 185, 6348-6354.

Ye, Z.J., Zhou, Q., Yin, W., Yuan, M.L., Yang, W.B., Xiang, F., Zhang, J.C., Xin, J.B., Xiong, X.Z., and Shi, H.Z. (2012a). Interleukin 22-producing $\mathrm{CD}^{+} \mathrm{T}$ cells in malignant pleural effusion. Cancer Lett 326, 23-32.

Ye, Z.J., Zhou, Q., Yin, W., Yuan, M.L., Yang, W.B., Xiong, X.Z., Zhang, J.C., and Shi, H.Z. (2012b). Differentiation and immune regulation of IL-9-producing $\mathrm{CD}^{+} \mathrm{T}$ cells in malignant pleural effusion. Am J Respir Crit Care Med 186, 1168-1179.

Ye, Z.J., Zhou, Q., Zhang, J.C., Li, X., Wu, C., Qin, S.M., Xin, J.B., and Shi, H.Z. (2011). CD $39^{+}$regulatory $\mathrm{T}$ cells suppress generation and differentiation of Th17 cells in human malignant pleural effusion via a LAP-dependent mechanism. Respir Res 12, 77.

Zheng, W., and Flavell, R.A. (1997). The transcription factor GATA-3 is necessary and sufficient for Th2 cytokine gene expression in CD4 T cells. Cell 89, 587-596.

Zhou, M., and Ouyang, W. (2003). The function role of GATA-3 in Th1 and Th2 differentiation. Immunol Res 28, 25-37.

Zhou, Y., Sonobe, Y., Akahori, T., Jin, S., Kawanokuchi, J., Noda, M., Iwakura, Y., Mizuno, T., and Suzumura, A. (2011). IL-9 promotes Th17 cell migration into the central nervous system via CC chemokine ligand-20 produced by astrocytes. J Immunol 186, 4415-4421.

Zou, W., and Restifo, N.P. (2010). $\mathrm{T}_{\mathrm{H}} 17$ cells in tumour immunity and immunotherapy. Nat Rev Immunol 10, 248-256.

Open Access This article is distributed under the terms of the Creative Commons Attribution License which permits any use, distribution, and reproduction in any medium, provided the original author(s) and source are credited. 